\title{
Falling through the black hole horizon
}

\author{
Ram Brustein $^{a}$ and A.J.M. Medved ${ }^{b, c}$ \\ ${ }^{a}$ Department of Physics, Ben-Gurion University, \\ Beer-Sheva 84105, Israel \\ ${ }^{b}$ Department of Physics $\&$ Electronics, Rhodes University, \\ Grahamstown 6140, South Africa \\ ${ }^{c}$ National Institute for Theoretical Physics (NITheP), \\ Matieland, Western Cape 7602, South Africa \\ E-mail: ramyb@bgu.ac.il, j.medved@ru.ac.za
}

ABSTRACT: We consider the fate of a small classical object, a "stick", as it falls through the horizon of a large black hole (BH). Classically, the equivalence principle dictates that the stick is affected by small tidal forces, and Hawking's quantum-mechanical model of BH evaporation makes essentially the same prediction. If, on the other hand, the $\mathrm{BH}$ horizon is surrounded by a "firewall", the stick will be consumed as it falls through. We have recently extended Hawking's model by taking into account the quantum fluctuations of the geometry and the classical back-reaction of the emitted particles. Here, we calculate the strain exerted on the falling stick for our model. The strain depends on the near-horizon state of the Hawking pairs. We find that, after the Page time when the state of the pairs deviates significantly from maximal entanglement (as required by unitarity), the induced strain in our semiclassical model is still parametrically small. This is because the number of the disentangled pairs is parametrically smaller than the BH entropy. A firewall does, however, appear if the number of disentangled pairs near the horizon is of order of the $\mathrm{BH}$ entropy, as implicitly assumed in previous discussions in the literature.

KeYwords: Models of Quantum Gravity, Black Holes

ARXIV EPRINT: 1503.05597 


\section{Contents}

1 Introduction $\quad 1$

1.1 A thought experiment and its outcome 3

1.2 Comparison with a previous analysis 4

2 The semiclassical model $\quad 5$

3 The Hawking modes near the horizon $\quad 6$

4 The fate of a falling stick $\quad 8$

4.1 Setup of the thought experiment 8

4.2 The stress-energy tensor of the disentangled modes 11

4.3 The curvature induced by the disentangled modes 12

4.3.1 Curvature from the stress-energy tensor $\quad 12$

4.3.2 Curvature from the shock wave approximation 13

4.4 The induced strain 13

4.5 The induced strain in different models 14

5 Summary $\quad 15$

\section{Introduction}

What would be the fate of a small classical object as it falls through the horizon of a large black hole $(\mathrm{BH})$ ? Given that the $\mathrm{BH}$ is large enough, classical relativity predicts that the object will only suffer a small tidal force. As Hawking's quantum-mechanical model of $\mathrm{BH}$ evaporation makes essentially the same prediction [1-3], this was long thought to be a settled matter. Nonetheless, the table has since been turned on account of the recent "firewall" proposal, which suggests that the object will rather be obliterated due to interactions with high-energy quanta [4]. Also see [5-10] for earlier, related discussions, [11-14] for important clarifications and [15-46] for what is just a sampling of the firewall literature.

We have recently developed a new semiclassical model of BH evaporation and would now like to find out what our model predicts for the fate of a falling object. We have so far studied the model both from the perspective of the emitted Hawking radiation as observed from outside the $\mathrm{BH}[47-50]$ and from the perspective of pair production near the $\mathrm{BH}$ horizon [51-53]. The premise has been to extend Hawking's original models respectively, the collapsing matter shell $[1,2]$ and the eternal $\mathrm{BH}[3]$ — in a way that incorporates the quantum fluctuations of the background geometry and the back-reaction effects of the produced pairs. 
The main idea of our semiclassical model is that one has to treat the $\mathrm{BH}$ as a quantum state [54] rather than a fixed classical geometry. This induces corrections that are nonperturbative from the perspective of an effective theory of quantum fields on a fixed curved background but, yet, can significantly alter the outcomes. The analysis is carried out by introducing a Gaussian wavefunction for the horizon of the (incipient) BH, as motivated in [54-57], and reevaluating all relevant quantities as expectation values. In effect, we take into account that the $\mathrm{BH}$ is of finite size and monotonically decaying throughout the process.

Here, we are mostly interested in the pair-production point of view. As discussed previously in $[51,52]$, we have realized a picture in which the produced pairs remain in the near-horizon zone a parametrically short time in comparison to the Page time $[58,59]$ (i.e., the midpoint of evaporation in units of entropy). After this briefer interval of time - which we have called the coherence time $t_{\text {coh }}$ - the negative-energy modes should be viewed as having been subsumed by the $\mathrm{BH}$ interior and their positive-energy partners, as having transitioned to the external Hawking radiation. Then, as a consequence of this continual depletion of modes from the near-horizon zone, the number of pairs in this region is of the order of the square root of the BH entropy.

This last outcome should, in a qualitative sense, really be regarded as generic. After all, given any model for which the $\mathrm{BH}$ mass is finite and decreasing in time due to the emission of particles, the number of pairs in the near-horizon region should be parametrically smaller than the BH entropy. For instance, by the Page time, about half of the particles that will ever be emitted by the BH have already moved far away from the horizon and transitioned into "real" Hawking particles. Clearly, then, the number of pairs in the zone cannot be any larger than the remaining number of would-be Hawking particles that are still waiting to be emitted.

We will show that this estimate for the number of pairs implies that they induce a parametrically small force on free-falling objects crossing the horizon. This is in direct contrast to the aforementioned firewall proposal.

The basic idea underlying the firewall proposal is as follows: the standard properties of quantum mechanics, such as unitary evolution and the strong subadditivity of entropy, prohibit the positive-energy modes in the zone from being concurrently entangled with both their negative-energy partners and the older, outgoing Hawking particles. The former is necessary to ensure that the horizon is free of drama, while the latter is needed for the eventual purification of the radiation. This conclusion is indeed correct and requires the produced pairs in our model to deviate significantly from maximal entanglement at times later than the Page time [53]. However, this observation by itself does not determine the number of disentangled pairs near the horizon and, hence, the amount of excitation in the near-horizon region above the Hartle-Hawking vacuum.

We have previously studied the plight of an in-falling shell of matter (quantum or classical) as it passes through the horizon [52]. Our findings revealed that the shell "sees" 1 excitations of the vacuum that are parametrically suppressed relative to the Planckian

\footnotetext{
${ }^{1}$ More accurately, what an external observer perceives the shell to see.
} 
energy scales that are normally attributed to a firewall. However, this analysis was limited in the following three ways: first, our calculation was based simply on estimating the magnitude of the energy density near the horizon. What really is needed would be a physical result that can be directly compared to the situation when no excited modes are present. Second, we would like to re-express the situation as much as possible from the direct perspective of the falling object. This is a non-trivial extension because our framework - just like Hawking's - is formulated from the perspective of an external, stationary observer. Third, we had not yet accounted for the possibility of strong deviations from maximal entanglement and the resulting properties of the state of the matter fields in the near-horizon zone. As it turns out, we only need some limited information about the near-horizon state and do not need to know the state of the interior of the $\mathrm{BH}$.

\subsection{A thought experiment and its outcome}

We are proposing a thought experiment that consists of dropping a cylindrical "stick" radially towards the $\mathrm{BH}$ horizon and asking how its journey is influenced by the disentangled modes within the near-horizon region. We can calculate the total number of pairs in this region and the degree of disentanglement amongst them. These inputs enable us to determine the curvature that is induced by the disentangled modes and then, by way of the geodesic deviation equation, the corresponding force on the stick in terms of a dimensionless parameter, the mechanical strain. Then we can discuss whether the induced strain can be used to detect the position of the horizon and to what extent, if at all, the falling stick is consumed as it falls through this surface.

The current approach allows us to discuss the geodesic deviation equation from the perspective of the falling stick. Hence, there is no longer any need to speculate as to what is the precise definition of the firewall, which remains elusive. We can compare the gravitational force delivered by the disentangled modes to that delivered by the background Riemann curvature and discuss the implications. This can be done for Hawking's model, for our semiclassical model and for the Page model $[58,59]$ as implicitly interpreted in the context of the firewall discussions. As for the force induced by additional interactions, such as those of electromagnetism, it is likely to be subdominant, but this issue should probably be considered in more detail.

We find that the exerted force is proportional to the number of disentangled modes in the vicinity of the horizon and to the amount of disentanglement. After the Page time, the amount of disentanglement per mode is of order unity and, consequently, the force is proportional to the number of Hawking pairs in the near-horizon region. We find for our model that the force delivered by the modes is parametrically larger than that of the background. On the other hand, the strain on the stick is still parametrically small - it is suppressed by the ratio of the length of the stick to the $\mathrm{BH}$ radius. This smallness can be attributed, once again, to the bounded number of pairs in the near-horizon region.

In the Page model $[58,59]$, as implicitly interpreted in the firewall discussions, the number of pairs becomes of order of the BH entropy at the Page time and the degree of disentanglement per mode grows to order unity by the same time [11]. As a result, the force on the stick becomes Planckian, inducing a parametrically large strain which is 
so large that the stick indeed breaks up. So that, in this case, we find a phenomenon whose outcome leads to a disintegration of the stick and could certainly be interpreted as a "firewall". In the Hawking model, on the other hand, the modes are always maximally entangled up to small corrections, and so their impact on the stick is much smaller than that of the background curvature.

In summary, the arguments for a firewall in [4] are basically substantiated by our results, since the near-horizon state has to be different than that which is predicted by an effective theory of fields on a fixed BH background. But, at least for our semiclassical model, the degree of deviation from the standard vacuum is much smaller than claimed.

\subsection{Comparison with a previous analysis}

The current treatment was motivated in part by that of Itzhaki [5], which can be viewed as the first realization of what only later was dubbed a BH firewall. Itzhaki posed the following question: what is the effect of a gravitational shock wave due to an outgoing Hawking mode on an ingoing test particle? This was computed and the answer was summed over all such shock waves that the test particle encounters on its way to the horizon. Itzhaki found that the net effect is to displace the particle so far from its original (null) trajectory that it never even has the opportunity to cross the horizon — the BH had already evaporated before the particle ever got there.

This is a remarkable finding and obviously a much different one than ours. But we believe that there is no contradiction. Itzhaki's conclusion is based on the exponential squeezing of the modes in the vicinity of the horizon; in other words, the exponentially large near-horizon redshift in Hawking's model. The result is that, before the test particle ever reaches the horizon, it crosses the path of all the emitted Hawking particles.

We, however, view this infinite redshift as an approximation of treating the background geometry as a strictly classical entity [57] (which is tantamount to assuming an infinitely massive $\mathrm{BH}$ ) and the test particle as strictly point like. In our framework, the quantum fluctuations of the $\mathrm{BH}$ regulate this would-be infinite redshift. In fact, as will be shown, the redshift is a "red herring" - the piling-up of modes near the horizon is mitigated by the continual depletion of incipient Hawking particles from the near-horizon zone, insofar as the redshift has been suitably regulated.

As we will also be discussed later, our proposal for the energy density of the disentangled modes is parametrically larger than in Itzhaki's model but, in spite of this difference, the induced gravitational interactions on a finite object are still small.

Contents. The rest of the paper is organized as follows: the next section contains a brief explanation of our semiclassical model of $\mathrm{BH}$ evaporation. Then, in section 3 , we use a novel physical argument to affirm our previous description of the pair-produced modes in [51]; namely, that the would-be Hawking particles "escape" from the near-horizon region after an interval of order $t_{\mathrm{coh}}$, which is parametrically shorter than the $\mathrm{BH}$ lifetime. The quantitative analysis of the induced strain is found in section 4, where we give a detailed account of the plight of the stick. Section 5 contains a brief summary. 


\section{The semiclassical model}

In the following, fundamental constants, besides the Planck length $l_{P}=\sqrt{\hbar G}$, are usually set to unity except when needed for clarity. We are mostly interested in parametric dependence and so typically neglect numerical factors.

We assume, for concreteness, a four-dimensional Schwarzschild BH with metric $d s^{2}=$ $-F(r) d u d v+r^{2} d \Omega^{2}$, where $F(r)=1-R_{S} / r$ and $R_{S}=2 l_{P}^{2} M$ is the Schwarzschild radius. Also, $u, v$ are the retarded and advanced null coordinates, $u, v=t \mp r_{*}$, such that $r_{*}=\int \frac{d r}{F(r)}$ is the Tortoise coordinate. The BH entropy is $S_{\mathrm{BH}}=\frac{\pi R_{S}^{2}}{l_{P}^{2}}$ and the $\mathrm{BH}$ is semiclassical, $S_{\mathrm{BH}} \gg 1$.

We use $N$ to denote either the cumulative number of particles emitted from the $\mathrm{BH}$ or the cumulative number of pairs produced (these are parametrically the same number) and $N_{\text {pairs }}$ to denote the number of pairs in the near-horizon zone at some given time.

Our semiclassical model is similar in many respects to the Hawking model of $\mathrm{BH}$ evaporation. However, there is a significant difference: the $\mathrm{BH}$ is treated as a quantum state and its quantum fluctuations are not neglected. In practice, we achieve this goal by assigning the (incipient) $\mathrm{BH}$ a Gaussian wavefunction $[54,56,57]$

$$
\Psi_{\mathrm{BH}}(R)=\mathcal{N}^{-1 / 2} e^{-\frac{\pi}{2 l_{P}^{2}}\left(R-R_{S}\right)^{2}},
$$

where $R$ parametrizes the fluctuating position of the quantum horizon and $\mathcal{N}$ is a normalization constant. We then calculate quantum expectation values rather than work directly with the classical metric. For an observable $\widehat{O}$, this means calculating

$$
\left\langle\Psi_{\mathrm{BH}}|\widehat{O}| \Psi_{\mathrm{BH}}\right\rangle=4 \pi \int_{0}^{\infty} d R R^{2} O(R) \Psi_{\mathrm{BH}}^{2}(R) .
$$

The small parameter in our model is the "classicality" parameter, $C_{\mathrm{BH}}=1 / S_{\mathrm{BH}}$. What is essentially the same parameter also appears in [60-64]. Technically, it is introduced by the width of $\Psi_{\mathrm{BH}}$. The fact that the classicality parameter does not vanish - it is rather small but finite - can result in modifications to physical quantities. The differences are most pronounced for quantities that are either vanishing or divergent in the classical limit $C_{\mathrm{BH}}=0$.

In anticipation of the upcoming sections, we list here several relevant results:

$$
\begin{aligned}
\left\langle\Psi_{\mathrm{BH}}\left|F^{2}\right| \Psi_{\mathrm{BH}}\right\rangle & \equiv \lim _{r \rightarrow R_{S}}\left\langle\Psi_{\mathrm{BH}}\left|\left(\frac{r-R}{R}\right)^{2}\right| \Psi_{\mathrm{BH}}\right\rangle \simeq S_{\mathrm{BH}}^{-1}, \\
\left\langle\Psi_{\mathrm{BH}}\left|F^{-2}\right| \Psi_{\mathrm{BH}}\right\rangle & \equiv \lim _{r \rightarrow R_{S}}\left\langle\Psi_{\mathrm{BH}}\left|\left(\frac{R}{r-R}\right)^{2}\right| \Psi_{\mathrm{BH}}\right\rangle \simeq S_{\mathrm{BH}}, \\
\left\langle\Psi_{\mathrm{BH}}\left|F^{-4}\right| \Psi_{\mathrm{BH}}\right\rangle & \equiv \lim _{r \rightarrow R_{S}}\left\langle\Psi_{\mathrm{BH}}\left|\left(\frac{R}{r-R}\right)^{4}\right| \Psi_{\mathrm{BH}}\right\rangle \simeq S_{\mathrm{BH}}^{2},
\end{aligned}
$$

where the latter two follow from the use of

$$
\int d x \frac{1}{x^{2 n}} e^{-S_{\mathrm{BH}} x^{2}} \simeq \Gamma\left(\frac{-n+1}{2}\right) S_{\mathrm{BH}}^{n} .
$$


An important time scale that accounts for the difference between an infinitely massive $\mathrm{BH}$ and one with a finite but large mass is the so-called coherence time. This time scale is typically $N_{\text {coh }} \simeq \sqrt{S_{\mathrm{BH}}}$ (which reads in Schwarzschild units as $t_{\mathrm{coh}} \simeq \frac{R_{S}^{2}}{l_{P}}$ ) and has a dual meaning: first, from the perspective of the external radiation, $N_{\text {coh }}$ is the scale of temporal extent for the matter correlations. This comes about because wavefunctions for the $\mathrm{BH}$ at different times are orthogonal when this time difference exceeds the coherence scale. Second, from the perspective of the produced pairs, $N_{\text {coh }}$ is the time that a mode stays in the near-horizon zone; after which, the negative-energy modes are subsumed by the interior matter and the positive-energy modes escape to become Hawking particles. It is latter meaning that is significant to the current work and will be substantiated in the section to follow.

This is the bare necessity that a reader needs to know about our model for BH evaporation. Our earlier, cited papers can be consulted for more comprehensive discussions.

\section{The Hawking modes near the horizon}

Here, we will reconsider the pair-production picture of $\mathrm{BH}$ evaporation. Many of the aspects are common to the Hawking and semiclassical models. We will emphasize these aspects as well as the differences as they turn up in the discussion.

If quantities are averaged over sufficiently long time periods, it should be clear that the average number of produced pairs must match the average number of emitted particles. On average, a Hawking particle is emitted once every Schwarzschild time $t \sim R_{S}$, and so pairs are produced at the same rate. Then, since $R_{S} \ll t_{\text {coh }}=R_{S} \frac{R_{S}}{l_{P}}$, we can treat both processes as acting continuously when looking at intervals of coherence time.

In this way, the process of $\mathrm{BH}$ evaporation entails the continuous production of pairs and the continuous absorption of negative-energy modes. Meanwhile, positive-energy modes are transitioning into the outgoing Hawking radiation as their subsumed negativeenergy partners are being absorbed into the interior matter, continually reducing the $\mathrm{BH}$ mass. All of these rates are determined, on average, by the BH's thermal rate of emission.

Let us begin the analysis from the perspective of a local, free-falling observer. We are interested only in the massless modes with low angular momenta, which eventually do escape from the near-horizon zone. These are the modes that are constrained by the arguments of strong subadditivity while the rest are in their vacuum states. Each of the massless modes will have a momentum of magnitude $E$, where $E \sim 1 / R_{S}$, as $R_{S}$ sets the size of the wavelength. It then follows, from momentum conservation and from Hawking's realization that the positive- and negative-energy partners are created (respectively) just outside and just inside of the horizon, that their momenta are initially of the form

$$
\begin{aligned}
& \vec{p}=\cos \alpha E \hat{Y}+\sin \alpha E \hat{U}, \\
& \vec{q}=-\cos \alpha E \hat{Y}-\sin \alpha E \hat{U},
\end{aligned}
$$

for the positive- and negative-energy mode respectively. Here, $\hat{Y}$ defines a direction along or on the horizon surface (it could be lightlike or spacelike), $\hat{U}$ is the lightlike Kruskal direction off the horizon and we are using the conventions that $U$ increases towards large values of $r$ and $0 \leq \alpha \leq \pi$. 
The unit vector $\hat{Y}$ is a linear combination of the unit vectors $\hat{\theta}, \hat{\phi}$ and the null Kruskal direction along the horizon $\hat{V}$. The exact form of this linear combination is not relevant to the current considerations. In most cases, $\sin \alpha,|\cos \alpha|$ are of order unity and will be dropped for now on.

The above relations are from a local, free-falling perspective. A stationary observer at large $r$ still detects modes with energy $E \sim 1 / R_{S}$ but sees the momentum in the $U$ direction as being red-shifted according to

$$
\begin{aligned}
& \vec{p}=E \hat{Y}+e^{-u / 2 R_{S}} E \hat{U}, \\
& \vec{q}=-E \hat{Y}-e^{-u / 2 R_{S}} E \hat{U},
\end{aligned}
$$

where $u$ is the retarded time coordinate and the redshift factors are meant to account for both the energy and the velocity of the mode.

Now, given the standard classical geometry of Hawking's model, $u \simeq-2 R_{S} \ln \left(\frac{r-R_{S}}{R_{S}}\right),{ }^{2}$ and then $e^{-u / 2 R_{S}} \simeq \frac{r-R_{S}}{R_{S}} \rightarrow 0$ as $r \rightarrow R_{S}$. This makes it clear that, from a stationaryobserver's viewpoint, the $U$ component of the momentum $\vec{P}_{U}$ vanishes and so the partners are forever trapped on the horizon. This is consistent with Hawking's description of the pair-production process in [3], as there an eternal $\mathrm{BH}$ spacetime is assumed.

But our semiclassical model leads to a different result. The average $\left\langle\Psi_{\mathrm{BH}}\left|\vec{p}_{U}\right| \Psi_{\mathrm{BH}}\right\rangle$ is still exponentially small; however the quantum fluctuations of the $\mathrm{BH}$ itself will lead to a small but finite variance [57]. Indeed, using the prescription (2.2) and the result (2.3), we find that

$$
\begin{aligned}
\left\langle\Psi_{\mathrm{BH}}\left|\vec{p}_{U} \cdot \vec{p}_{U}\right| \Psi_{\mathrm{BH}}\right\rangle & =E^{2}\left\langle\Psi_{\mathrm{BH}}\left|F^{2}\right| \Psi_{\mathrm{BH}}\right\rangle \\
& =\frac{E^{2}}{S_{\mathrm{BH}}}+\mathcal{O}\left[S_{\mathrm{BH}}^{-2}\right] .
\end{aligned}
$$

Now, since the average value of $\vec{p}_{U}$ is exponentially small and these modes are outgoing so that $\vec{p}_{U}$ positive by definition, we can use $\sqrt{\left\langle\left|\vec{p}_{U} \cdot \vec{p}_{U}\right|\right\rangle} \simeq E \frac{l_{p}}{R_{S}}$ as an estimate for the velocity of a mode in the direction orthogonal to the horizon,

$$
v_{U}=\frac{\sqrt{\left\langle\left|\vec{p}_{U} \cdot \vec{p}_{U}\right|\right\rangle}}{E} \simeq \frac{l_{p}}{R_{S}}
$$

We can then quantify the time of escape by using the above estimate. It follows that the time a Hawking mode takes to reach a distance $R_{S}$ away from the horizon is given by

$$
t_{\text {escape }} \simeq \frac{R_{S}}{v_{U}} \simeq R_{S} \frac{R_{S}}{l_{P}}=t_{\text {coh }} .
$$

In effect, the normally divergent factor in the escape-time estimate has been replaced by the large but finite factor $R_{S} / l_{P}$. To summarize, the effect of the quantum fluctuations is to make the redshift finite - it takes the outgoing mode a finite time to escape the near-horizon region.

\footnotetext{
${ }^{2}$ The factor of 2 is because $t \sim-r_{*}$ at the future horizon.
} 
The number of actively entangled pairs $N_{\text {pairs }}$ is then of the same order as the number of pairs that are produced by the $\mathrm{BH}$ over a time period $t_{\text {coh }}$; i.e., $N_{\text {pairs }} \sim N_{\text {coh. }}$. As commented upon earlier and detailed elsewhere [51, 52], this truncation in the number of partnered modes - from order $S_{\mathrm{BH}}$ to order $N_{\mathrm{coh}}=S_{\mathrm{BH}}^{1 / 2}-$ is a critical part of our argument for resolving the aforementioned firewall problem.

Another important quantity that we would like to introduce is $N_{\text {dis }}$, the number of active pairs times the degree of disentanglement per pair $\mathcal{D}_{\text {dis }}$. This is a model-dependent outcome, as it requires a specification of the state of the pairs or, at the very least, some means of quantifying how much this state deviates from the Hartle-Hawking state of maximal entanglement. Let us first recall that, for our model, $N_{\text {pairs }}$ is bounded from above by $N_{\text {coh }}=\sqrt{S_{\mathrm{BH}}}$ and so $N_{\text {dis }} \lesssim \sqrt{S_{\mathrm{BH}}}$.

In addition, we have recently [53] found a means for estimating $\mathcal{D}_{\text {dis }}$ in our framework. By partitioning the system of Hawking modes into three subsystems - the already emitted Hawking particles (or early radiation) $A$, the positive-energy modes in the zone (or late radiation) $B$ and their negative-energy partners $C$ - we have evaluated the entanglement between $A$ and $B .{ }^{3}$ The condition of strong subadditivity of entropy then enforces a lower bound on the the degree of disentanglement between a pair of modes in $B$ and $C$. The need for such a bound is quite natural, given that "monogamy of entanglement" is in play and that any positive-energy mode in the zone must have some degree of entanglement with subset $A$ if the state of the radiation is to eventually purify. What the analysis in [53] does is put this idea on a more quantitative level.

And so, taking this lower bound as an estimate for the degree of disentanglement per pair, we have

$$
\mathcal{D}_{\text {dis }} \simeq \frac{N C_{\mathrm{BH}}-1}{1+N C_{\mathrm{BH}}}
$$

One can notice that $\mathcal{D}_{\text {dis }}$ depends solely on the product $N C_{\mathrm{BH}}=N(t) / S_{\mathrm{BH}}(t)$, which happens to be the effective perturbative parameter for our framework [47]. One can also see that, after the Page time when $N C_{\mathrm{BH}} \geq 1$, the amount of disentanglement is of order unity, $\mathcal{D}_{\text {dis }} \sim 1$.

As a lower bound, this estimate is not useful at times before the Page time, for which $\mathcal{D}_{\text {dis }}$ is negative. However, when the $\mathrm{BH}$ is still young, $\mathcal{D}_{\text {dis }}$ must be a parametrically small number, as any model of BH evaporation should reduce to Hawking's model plus perturbatively small corrections at such early times. We will always be assuming that the $\mathrm{BH}$ is older than the Page time, as this is the regime of interest as far as the prospects for a firewall are concerned [4].

\section{The fate of a falling stick}

\subsection{Setup of the thought experiment}

We will next consider the consequences of our framework for a stick falling through the horizon of a semiclassical BH. By stick, we mean a classical, cylindrical object of length $\ell$

\footnotetext{
${ }^{3}$ We used Renyi entropies for the analysis, with this choice justified in [53].
} 


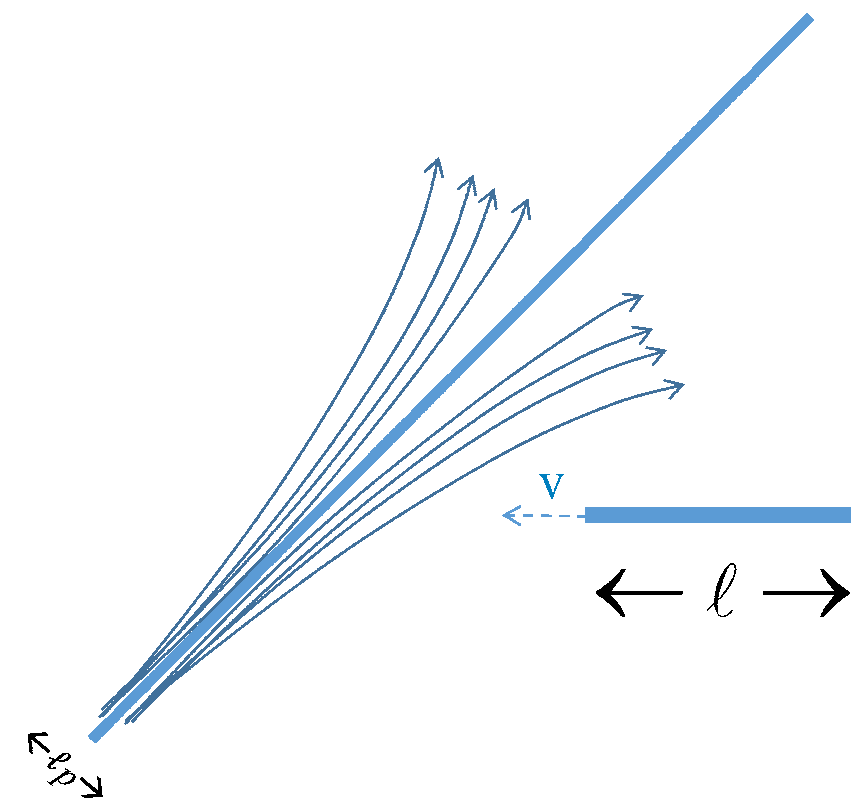

Figure 1. Spacetime diagram showing the Hawking pairs near the BH horizon with the falling stick away from the horizon.

and radius $s$, such that $l_{P} \ll s \lesssim \ell \ll R_{S}$. The local frame of the stick will be denoted by $T, X, Y$ and $Z$. It is assumed to be falling toward the horizon on a radial trajectory with its long side aligned parallel to the direction of motion - decreasing $r$ or, locally for the stick, decreasing $X$. In figure 1 , the falling stick is depicted in a space-time diagram.

Before proceeding, we need to make sure that the stick does not disintegrate due to any tidal forces arising from the gravitational background. This requirement imposes a constraint on the ratio $\ell / R_{S}$; i.e., the ratio of the size of the stick to the Schwarzschild radius of the $\mathrm{BH}$. This comes about because the stick will experience a relative longitudinal acceleration between its ends, for which the magnitude near the horizon is given by $\Delta a \sim \frac{G M}{R_{S}^{2}} \frac{\ell}{R_{S}}$.

To continue with this idea, let us assume that the stick is made of some elastic material; then $\Delta a \sim \frac{K}{\rho} \Delta \ell$, where $\rho$ is the mass density of the stick and $K$ is its bulk modulus. It follows that, near the horizon, $\frac{K}{\rho} \Delta \ell \sim \frac{G M}{R_{S}^{2}}\left(\ell / R_{S}\right)$ or $\Delta \ell / \ell \sim(\rho / K)\left(c^{2} / R_{S}^{2}\right)$. Here, we have reinstated the speed of light $c$ and used that $R_{S}=2 G M / c^{2}$. But $(K / \rho) \sim \omega_{\text {stick }}^{2} \sim$ $\left(c_{\text {sound }} / \ell\right)^{2}$, and so the result is $\Delta \ell / \ell \sim\left(c / c_{\text {sound }}\right)^{2}\left(\ell / R_{S}\right)^{2}$. Meaning that, if we insist upon $\Delta \ell / \ell<1$, then $\ell / R_{S}<c_{\text {sound }} / c$. For known materials, this ratio is no larger than about $10^{-4}$. We can then conclude that, to avoid the breaking up of the stick, the ratio $\ell / R_{S}$ has to be parametrically small.

For this setup, the acceleration of the stick in the $Y$ (or $Z$ ) direction can be determined from the geodesic deviation equation for Einstein's gravity. What we want to know, in particular, is the induced gravitational force which is delivered to the stick by the disentangled Hawking modes near the horizon. This force will eventually be parametrized in terms of a dimensionless scalar quantity, the deformation per unit length or the strain $\gamma$. 


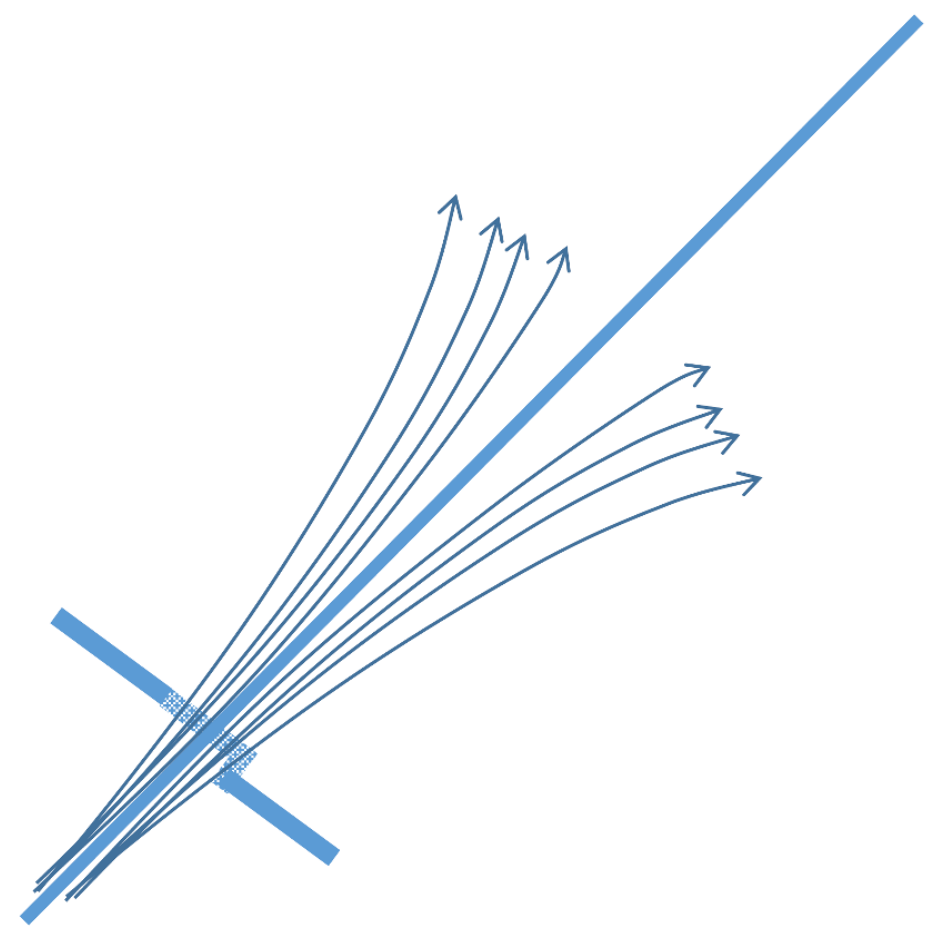

Figure 2. Spacetime diagram showing the deformation of the stick induced by the disentangled Hawking modes as it crosses the horizon.

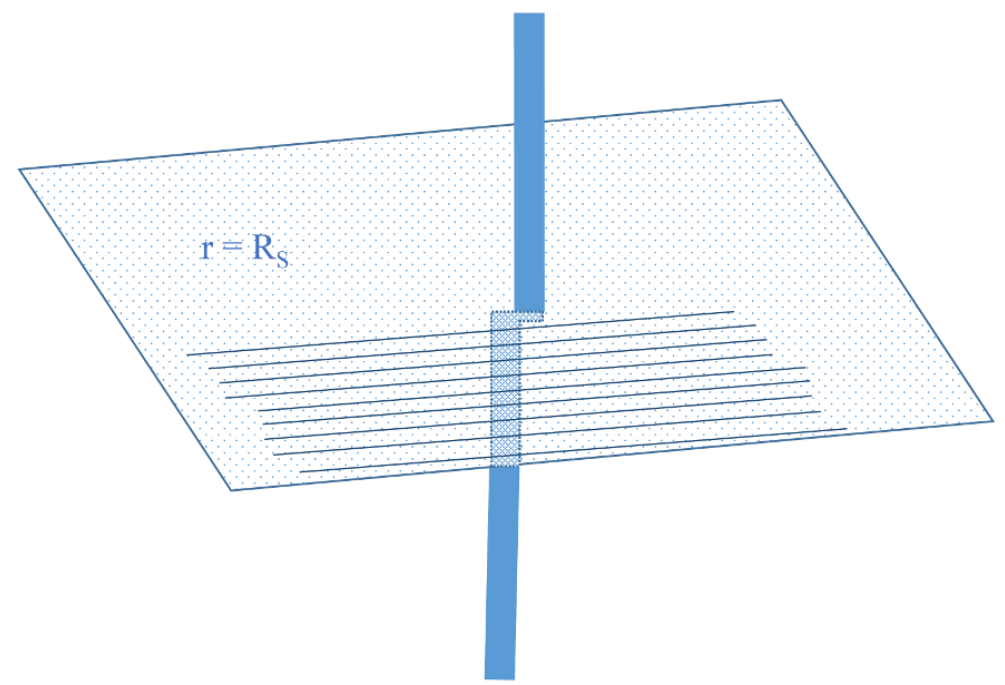

Figure 3. The deformation of the stick induced by the disentangled Hawking modes across the surface $r=R_{S}$.

Let us pause to comment briefly on the physical picture. Far away from the horizon - where the Hawking modes are dilute and their induced force is weak - the net force exerted on the stick will be negligible. Near the horizon, the situation is different. The effect of induced gravity is still relatively weak, but the part of the stick that is closer to the 
horizon will feel a stronger force than that which is farther. This is because the Hawking modes become both denser in number and more energetic as the horizon is approached. It is this gradient and the accompanying tidal force that could cause the stick to endure harm.

What we first need to know is the stress-energy tensor for the disentangled modes, from which Einstein's equation will give us the associated curvature and then the geodesic deviation equation will yield the tidal force. Alternatively, one can deduce the induced change to the metric by treating the Hawking modes as shock waves [5].

\subsection{The stress-energy tensor of the disentangled modes}

Knowledge of the stress tensor requires one to know about the number of disentangled modes $N_{\text {dis }}$ and the energy density of each of these. A detailed discussion about $N_{\text {dis }}$ will be deferred until later. We will determine the stress-energy tensor with respect to an external (stationary) observer's perspective, but the associated Riemann tensor will be extracted from a manifestly scalar quantity. In this way, the remainder of the calculation can proceed from the stick's own point of view.

A simple dimensional analysis suggests that the energy density of a fully disentangled mode is

$$
\varepsilon \simeq \frac{1}{R_{S}^{4} F}
$$

This expression formally diverges near the horizon; however, the fluctuations of the background regulate the divergence in a similar manner to the way in which the stretched horizon does. The reasoning for the estimate for $\varepsilon$ is as follows: according to an external observer, the modes are, up to red-shifting effects, delocalized over a spherical shell of radius $R_{S}$ and width $R_{S}$, while the energy scale of any given mode is set by the Hawking temperature, $1 / R_{S}$. Hence, the energy density per mode when the redshift is disregarded is $1 / R_{S}^{4}$. The Tolman redshift introduces a factor of $F(r)$ into the denominator, as both the inverse of the energy scale and the width of the shell are suppressed by a factor of $\sqrt{F(r)}$.

It then follows that

$$
\delta T_{u u} \sim \delta T_{v v} \sim \frac{N_{\text {dis }}}{R_{S}^{4} F}
$$

describes the energy flux for the disentangled modes as far as an external observer is concerned.

We also need to know the background stress tensor as would be measured by the same external observer and, for this, employ the standard Schwarzschild result from [65]. Then, very near to the horizon, $T_{u u} \sim T_{u v} \sim 0$ whereas $T_{v v} \sim-1 / R_{S}^{4}$. As each of these is parametrically much smaller than the estimate in eq. (4.2) for any $N_{\text {dis }}>l_{P} / R_{S}$, the background tensor can be disregarded in the subsequent analysis.

The estimate in eq. (4.2) can be compared to that of Itzhaki [5]. Working with Kruskal coordinates (for which $U \sim-R_{S} e^{-u / 2 R_{S}}$ and $V \sim R_{S} e^{v / 2 R_{S}}$ ), he proposed that $T_{U U} \sim \frac{l_{p}^{2}}{U^{2}}$ and $T_{V V} \sim 0$ near the horizon. But, recalling that $T_{u u} \sim U^{2} T_{U U}$ and $T_{V V} \sim T_{v v}$, we see that our proposal for $T_{u u}$ and $T_{v v}$ are both a factor of $1 / F$ more divergent at the horizon than their counterparts in [5], as well as those of the standard Hartle-Hawking and Unruh states. This can be attributed to the disentangled modes for our picture being highly 
concentrated in the proximity of the horizon, as per the previous section. From our point of the view, the modes further removed from the horizon have already "escaped".

\subsection{The curvature induced by the disentangled modes}

\subsubsection{Curvature from the stress-energy tensor}

We next want to convert Equation (4.2) into a statement about curvature and, as already stated, work with a scalar quantity. The simplest choice of scalar is

$$
G^{a b} G_{a b}=l_{P}^{4} T^{a b} T_{a b} \simeq l_{P}^{4} g^{u v} g^{u v} \delta T_{v v} \delta T_{u u}
$$

where $G_{a b}$ is the Einstein tensor. The equality on the left follows from Einstein's equation and the relation on the right follows from the disentangled modes being the dominant source.

In our semiclassical framework, the expression on the far right should be regarded as an expectation value with respect to the $\mathrm{BH}$ wavefunction; this being the context in which the disentangled modes are revealed. What we are then calculating is the expectation value of the scalar $G^{a b} G_{a b}$ with respect to the same wavefunction, and so it is more appropriate to write

$$
\begin{aligned}
\left\langle\Psi_{\mathrm{BH}}\left|G^{a b} G_{a b}\right| \Psi_{\mathrm{BH}}\right\rangle & =l_{P}^{4}\left\langle\Psi_{\mathrm{BH}}\left|g^{u v} g^{u v} \delta T_{v v} \delta T_{u u}\right| \Psi_{\mathrm{BH}}\right\rangle \\
& \simeq N_{\mathrm{dis}}^{2} \frac{l_{P}^{4}}{R_{S}^{8}}\left\langle\Psi_{\mathrm{BH}}\left|\frac{1}{F^{4}}\right| \Psi_{\mathrm{BH}}\right\rangle \\
& \simeq \frac{N_{\mathrm{dis}}^{2}}{R_{S}^{4}},
\end{aligned}
$$

where the middle line follows from eq. (4.2) and the last line follows from taking the nearhorizon limit along with eq. (2.5).

One can now get a first hint about the fate of the falling stick by looking at various possibilities for $N_{\text {dis }}$. Clearly, the largest possible value for $N_{\text {dis }}$ is $S_{\mathrm{BH}}$. This assumes that a finite fraction of all the modes that were ever emitted by the $\mathrm{BH}$ remain in the vicinity of the horizon. In this case, we find from eq. (4.4) that $\left\langle G^{a b} G_{a b}\right\rangle_{\Psi_{\mathrm{BH}}} \sim \frac{1}{l_{P}^{4}}$, and so it is likely that the stick disintegrates before it reaches the horizon. But, for our semiclassical model and for an old-enough BH, $N_{\text {dis }} \sim N_{\text {pairs }} \sim \sqrt{S_{\mathrm{BH}}}$, for which $\left\langle G^{a b} G_{a b}\right\rangle_{\Psi_{\mathrm{BH}}} \sim \frac{1}{R_{S}^{2} l_{P}^{2}}$. Of course, if $N_{\text {dis }}$ is of order unity, then $\left\langle G^{a b} G_{a b}\right\rangle_{\Psi_{\mathrm{BH}}} \sim \frac{1}{R_{S}^{4}}$, which cannot be distinguished from the background curvature.

Let us next observe that, because of the Ricci scalar contribution in the Einstein tensor $G^{a}{ }_{b}=R_{b}^{a}-\frac{1}{2} R g^{a}{ }_{b}$, all diagonal components of the (induced) Riemann tensor will be of roughly the same magnitude; meaning that the diagonal components of $R_{b}^{a}$ will scale with $N_{\text {dis }} / R_{S}^{2}$. It can then be deduced that, in the stick's own frame where the metric is regular, the root-mean-square (RMS) value of the Ricci curvature is given by

$$
R_{B}^{A} \simeq \frac{N_{\text {dis }}}{R_{S}^{2}} \delta_{B}^{A},
$$

for $A, B=\{T, X, Y, Z\}$. One can immediately see that $R_{B}^{A}$ vanishes for the Hawking model (up to the implied background contribution) since $N_{\text {dis }}=0$ must be true in this case. 


\subsubsection{Curvature from the shock wave approximation}

There is another way to quantify the effect of the disentangled modes acting on the stick. This would be, following Itzhaki [5], to treat the modes as shock waves and estimate the change in the metric and the curvature due to the waves crossing the stick. It is appropriate to use Kruskal coordinates for this calculation if it is to be from the stick's own perspective. A shock wave of energy $E \sim 1 / R_{S}$ propagating outwards along the ray $U=U_{0}$ will change $g_{U U}$ by an amount $\delta g_{U U} \sim G E \delta\left(U-U_{0}\right) \sim l_{P}^{2} / R_{S} \delta\left(U-U_{0}\right)$. The large Tolman blueshift for the modes in our model can be incorporated by the estimate $\delta\left(U-U_{0}\right) \sim 1 / U$, with $U$ meant to be within a few Planck lengths from the horizon where $U \sim 0$. Then the total displacement will go as $\delta g_{U U} \sim N_{\text {dis }} l_{P}^{2} / R_{S} U$ (and a similar contribution to $\delta g_{V V}$ for the inward-moving modes). It can be verified that the deformed metric induces a near-horizon curvature of order

$$
R_{U}^{V} \sim-\frac{1}{R_{S}} \frac{\partial \delta g_{U U}}{\partial U} \sim N_{\text {dis }} \frac{l_{P}^{2}}{R_{S}^{2}} \frac{1}{U^{2}}
$$

and similarly for $R_{V}^{U}$. Then, since $U \sim R_{S} F$, we may use eq. (2.4) to conclude that the RMS value of the Ricci tensor is given by

$$
R_{U}^{V} \sim \frac{N_{\text {dis }}}{R_{S}^{2}}
$$

which is in perfect agreement with the estimate from eq. (4.5).

\subsection{The induced strain}

Let us next recall the geodesic deviation equation and apply it to the current setup,

$$
\frac{d^{2}(\Delta x)^{A}}{d \tau^{2}}=R_{C D}^{A B} V_{B} V^{C}(\Delta x)^{D},
$$

where $(\Delta x)^{D}$ describes the spatial extent of stick - so that $(\Delta x)^{X}=\ell,(\Delta x)^{Y}=(\Delta x)^{Z}=$ $s-$ and $V^{A}$ is the velocity vector for the stick in terms of proper time $\tau$.

Using that the velocity vector for the stick is $V^{A}=-\beta \delta^{A}{ }_{X}$ for some $\beta<1$, we have

$$
\begin{aligned}
\frac{d^{2}(\Delta x)^{A}}{d \tau^{2}} & =\beta^{2} R^{A X}{ }_{X D}(\Delta x)^{D} \\
& \left.\simeq \beta^{2} R_{D}^{A}(\Delta x)^{D}\right|_{A, D \neq X},
\end{aligned}
$$

where the second line follows from $R^{A X}{ }_{B X}$ being the same order as $R_{B}^{A}$.

Next, substituting eq. (4.5) for the Ricci tensor, we obtain

$$
\left.\frac{d^{2}(\Delta x)^{A}}{d \tau^{2}} \simeq \beta^{2} \frac{N_{\mathrm{dis}}}{R_{S}^{2}}(\Delta x)^{A}\right|_{A \neq X}
$$

or, after integrating twice,

$$
\frac{\delta(\Delta Y)}{\Delta Y} \simeq(\Delta \tau)^{2} \beta^{2} \frac{N_{\mathrm{dis}}}{R_{S}^{2}}
$$

and similarly for $Z$. Here, $\delta(\Delta Y)$ means the RMS deformation of the stick in the $Y$ direction, so that the magnitude of the left-hand side is the strain $\gamma$. 
At any given time, only a small (about Planck-sized) segment of the stick is exposed to the potentially dangerous near-horizon modes. For this reason, it is appropriate to start with the force acting on a segment of length $\Delta X \sim \Delta$ with $\Delta \gtrsim l_{P}$. The induced deformation on the stick is depicted in a spacetime diagram in figure 2 and at a fixed time in figure 3.

The proper time that it takes this segment to pass through the near-horizon zone is then $\Delta \tau \sim \Delta / \beta$. Given these inputs, eq. (4.11) translates into

$$
\gamma_{\Delta} \simeq N_{\mathrm{dis}} \frac{\Delta^{2}}{R_{S}^{2}}
$$

To estimate the total strain endured by the stick, we will assume the "worst-case scenario", in which the individual deformations add coherently. Then the total strain is simply $\ell / \Delta \gtrsim \ell / l_{P}$ times the previous result,

$$
\gamma_{\text {stick }} \lesssim N_{\text {dis }} \frac{l_{P} \ell}{R_{S}^{2}}
$$

The actual strain will depend on the whether or not the stick oscillates, its speed of sound and so forth. For instance, a more realistic estimate might rather be to add in quadrature the strains on each part of the stick. Then the result would be the RMS value $\gamma_{\text {stick }} \sim$ $\sqrt{\frac{\ell}{\Delta}\left(\gamma_{\Delta}\right)^{2}}$, but this (or any other) modification would only weaken the previous estimate.

\subsection{The induced strain in different models}

Let us start with the case which is implicitly based on our previous attempt [52] at quantifying the effects of firewall. There, we incorrectly estimated the disentanglement per mode $\mathcal{D}_{\text {dis }}$ as being equal to the product $N_{\text {coh }} C_{\mathrm{BH}} \ll 1$. Then $N_{\text {dis }}<1$ and the strain on the stick is

$$
\gamma_{\text {stick }} \simeq \frac{\ell l_{P}}{R_{S}^{2}} \ll 1 \quad\left[N_{\text {dis }}<1\right]
$$

which is vanishing in the classical limit. This outcome explains the underestimation in our previous study.

Let us now consider what happens in our semiclassical model when the more accurate estimate of $\mathcal{D}_{\text {dis }}$ in eq. (3.8) is utilized. Then, after the Page time, $\mathcal{D}_{\text {dis }} \simeq 1$ and so $N_{\text {dis }} \sim N_{\text {pairs }} \sim \sqrt{S_{\mathrm{BH}}} \sim \frac{R_{S}}{l_{P}}$. It follows that

$$
\gamma_{\text {stick }} \simeq \frac{\ell}{R_{S}} \ll 1 \quad\left[N_{\text {dis }} \sim \sqrt{S_{\mathrm{BH}}}\right] .
$$

This is clearly a small number, but how small? As we have seen before, if this experiment is to be conducted in a reasonable way, then this ratio is constrained by $\ell / R_{S}<c_{\text {sound }} / c \lesssim 10^{-4}$. So that, in this case, despite the fact that the energy density is parametrically larger than $1 / R_{S}^{4}$, the physical effect on the stick is still remarkably small. In other words, the equivalence principle is preserved.

Finally, let us consider the Page model, as interpreted in the context of the firewall problem and, in particular, after the Page time. The usual interpretation of the Page model 
is that the number of pairs near the horizon is limited only by the original $\mathrm{BH}$ entropy and each of these has order one disentanglement [11]. Hence, $N_{\mathrm{dis}} \sim S_{\mathrm{BH}}$ and one then obtains

$$
\gamma_{\text {stick }} \simeq \frac{\ell}{l_{P}} \gg 1 \quad\left[N_{\text {dis }} \sim S_{\mathrm{BH}}\right] .
$$

Such a large strain indicates that the stick is obliterated on its journey through the nearhorizon region. This outcome can best be viewed as further evidence that, given the assumptions of [4], a firewall is indeed an inevitable consequence.

\section{Summary}

Using a simple thought experiment, we have investigated how the fate of an in-falling classical object passing through the horizon depends on the state of the near-horizon Hawking radiation. We verified that our semiclassical framework for BH evaporation and pair production does not lead to a conflict with the equivalence principle of general relativity (while being consistent with standard quantum theory [53]). In particular, it was shown that, as long as the experiment of dropping an object through the near-horizon region can be safely carried out, the disentangled Hawking modes will do nothing further to jeopardize the serenity of the journey. This is true in spite of the disentanglement per mode being of order unity, as required for information to escape from the $\mathrm{BH}$, and can be attributed to the Hawking pairs having an effective lifetime that is parametrically smaller than the Page time. On the other hand, the Page model, as normally interpreted in the firewall literature, does lead to a conflict with the equivalence principle, thus substantiating the arguments of [4] and others.

\section{Acknowledgments}

We thank Sunny Itzhaki for many useful discussions and insights. The research of RB was supported by the Israel Science Foundation grant no. 239/10. The research of AJMM received support from an NRF Incentive Funding Grant 85353, an NRF Competitive Programme Grant 93595 and Rhodes Research Discretionary Grants. AJMM thanks Ben Gurion University for their hospitality during his visit.

Open Access. This article is distributed under the terms of the Creative Commons Attribution License (CC-BY 4.0), which permits any use, distribution and reproduction in any medium, provided the original author(s) and source are credited.

\section{References}

[1] S.W. Hawking, Black hole explosions, Nature 248 (1974) 30 [INSPIRE].

[2] S.W. Hawking, Particle Creation by Black Holes, Commun. Math. Phys. 43 (1975) 199 [INSPIRE].

[3] S.W. Hawking, Breakdown of Predictability in Gravitational Collapse, Phys. Rev. D 14 (1976) 2460 [InSPIRE]. 
[4] A. Almheiri, D. Marolf, J. Polchinski and J. Sully, Black Holes: Complementarity or Firewalls?, JHEP 02 (2013) 062 [arXiv: 1207.3123] [INSPIRE].

[5] N. Itzhaki, Is the black hole complementarity principle really necessary?, hep-th/9607028 [INSPIRE].

[6] S.D. Mathur, What Exactly is the Information Paradox?, Lect. Notes Phys. 769 (2009) 3 [arXiv: 0803.2030] [INSPIRE].

[7] S.D. Mathur, The information paradox: A pedagogical introduction, Class. Quant. Grav. 26 (2009) 224001 [arXiv:0909.1038] [INSPIRE].

[8] S.D. Mathur, What the information paradox is not, arXiv:1108.0302 [INSPIRE].

[9] S.L. Braunstein, S. Pirandola and K. Życzkowski, Better Late than Never: Information Retrieval from Black Holes, Phys. Rev. Lett. 110 (2013) 101301 [arXiv:0907.1190] [INSPIRE].

[10] S.L. Braunstein and S. Pirandola, Post-firewall paradoxes, arXiv:1411.7195 [INSPIRE].

[11] D. Marolf and J. Polchinski, Gauge/Gravity Duality and the Black Hole Interior, Phys. Rev. Lett. 111 (2013) 171301 [arXiv: 1307.4706] [INSPIRE].

[12] R. Bousso, Firewalls from double purity, Phys. Rev. D 88 (2013) 084035 [arXiv:1308.2665] [INSPIRE].

[13] R. Bousso, Violations of the Equivalence Principle by a Nonlocally Reconstructed Vacuum at the Black Hole Horizon, Phys. Rev. Lett. 112 (2014) 041102 [arXiv: 1308.3697] [InSPIRE].

[14] S.D. Mathur, What does strong subadditivity tell us about black holes?, Nucl. Phys. Proc. Suppl. 251-252 (2014) 16 [arXiv: 1309.6583] [INSPIRE].

[15] L. Susskind, Singularities, Firewalls and Complementarity, arXiv:1208.3445 [INSPIRE].

[16] L. Susskind, The Transfer of Entanglement: The Case for Firewalls, arXiv:1210.2098 [INSPIRE].

[17] R. Bousso, Complementarity Is Not Enough, Phys. Rev. D 87 (2013) 124023 [arXiv:1207.5192] [INSPIRE].

[18] Y. Nomura, J. Varela and S.J. Weinberg, Complementarity Endures: No Firewall for an Infalling Observer, JHEP 03 (2013) 059 [arXiv:1207.6626] [INSPIRE].

[19] Y. Nomura, J. Varela and S.J. Weinberg, Black Holes, Information and Hilbert Space for Quantum Gravity, Phys. Rev. D 87 (2013) 084050 [arXiv:1210.6348] [InSPIRE].

[20] Y. Nomura, J. Varela and S.J. Weinberg, Black Holes or Firewalls: A Theory of Horizons, Phys. Rev. D 88 (2013) 084052 [arXiv: 1308.4121] [INSPIRE].

[21] S.D. Mathur and D. Turton, Comments on black holes I: The possibility of complementarity, JHEP 01 (2014) 034 [arXiv:1208.2005] [INSPIRE].

[22] A. Giveon and N. Itzhaki, String Theory Versus Black Hole Complementarity, JHEP 12 (2012) 094 [arXiv: 1208.3930] [INSPIRE].

[23] S.G. Avery, B.D. Chowdhury and A. Puhm, Unitarity and fuzzball complementarity: 'Alice fuzzes but may not even know it!', JHEP 09 (2013) 012 [arXiv:1210.6996] [INSPIRE].

[24] K. Larjo, D.A. Lowe and L. Thorlacius, Black holes without firewalls, Phys. Rev. D 87 (2013) 104018 [arXiv:1211.4620] [INSPIRE]. 
[25] E. Verlinde and H. Verlinde, Black Hole Entanglement and Quantum Error Correction, JHEP 10 (2013) 107 [arXiv: 1211.6913] [INSPIRE].

[26] E. Verlinde and H. Verlinde, Passing through the Firewall, arXiv:1306.0515 [InSPIRE].

[27] E. Verlinde and H. Verlinde, Black Hole Information as Topological Qubits, arXiv: 1306.0516 [INSPIRE].

[28] E. Verlinde and H. Verlinde, Behind the Horizon in AdS/CFT, arXiv:1311.1137 [INSPIRE].

[29] K. Papadodimas and S. Raju, An Infalling Observer in AdS/CFT, JHEP 10 (2013) 212 [arXiv:1211.6767] [INSPIRE].

[30] K. Papadodimas and S. Raju, Black Hole Interior in the Holographic Correspondence and the Information Paradox, Phys. Rev. Lett. 112 (2014) 051301 [arXiv:1310.6334] [InSPIRE].

[31] K. Papadodimas and S. Raju, State-Dependent Bulk-Boundary Maps and Black Hole Complementarity, Phys. Rev. D 89 (2014) 086010 [arXiv:1310.6335] [InSPIRE].

[32] S.B. Giddings, Nonviolent nonlocality, Phys. Rev. D 88 (2013) 064023 [arXiv:1211.7070] [INSPIRE].

[33] S.B. Giddings, Nonviolent information transfer from black holes: A field theory parametrization, Phys. Rev. D 88 (2013) 024018 [arXiv:1302.2613] [INSPIRE].

[34] T. Jacobson, Boundary unitarity and the black hole information paradox, Int. J. Mod. Phys. D 22 (2013) 1342002 [arXiv: 1212.6944] [INSPIRE].

[35] D. Harlow and P. Hayden, Quantum Computation vs. Firewalls, JHEP 06 (2013) 085 [arXiv: 1301.4504] [INSPIRE].

[36] S.G. Avery and B.D. Chowdhury, Firewalls in AdS/CFT, JHEP 10 (2014) 174 [arXiv: 1302.5428] [INSPIRE].

[37] A. Almheiri, D. Marolf, J. Polchinski, D. Stanford and J. Sully, An Apologia for Firewalls, JHEP 09 (2013) 018 [arXiv: 1304.6483] [INSPIRE].

[38] D.A. Lowe and L. Thorlacius, Pure states and black hole complementarity, Phys. Rev. D 88 (2013) 044012 [arXiv: 1305.7459] [INSPIRE].

[39] J. Maldacena and L. Susskind, Cool horizons for entangled black holes, Fortsch. Phys. 61 (2013) 781 [arXiv:1306. 0533] [INSPIRE].

[40] D.N. Page, Excluding Black Hole Firewalls with Extreme Cosmic Censorship, JCAP 06 (2014) 051 [arXiv: 1306. 0562] [INSPIRE].

[41] S.D. Mathur and D. Turton, The flaw in the firewall argument, Nucl. Phys. B 884 (2014) 566 [arXiv: 1306.5488] [INSPIRE].

[42] M. Van Raamsdonk, Evaporating Firewalls, JHEP 11 (2014) 038 [arXiv:1307.1796] [INSPIRE].

[43] S.L. Braunstein and S. Pirandola, Evaporating black holes have leaky horizons or exotic atmospheres, arXiv:1311.1326 [INSPIRE].

[44] S.L. Braunstein and S. Pirandola, Post-firewall paradoxes, arXiv:1411.7195 [INSPIRE].

[45] S.W. Hawking, Information Preservation and Weather Forecasting for Black Holes, arXiv:1401.5761 [INSPIRE]. 
[46] D. Harlow, Aspects of the Papadodimas-Raju Proposal for the Black Hole Interior, JHEP 11 (2014) 055 [arXiv: 1405.1995] [INSPIRE].

[47] R. Brustein and A.J.M. Medved, Restoring predictability in semiclassical gravitational collapse, JHEP 09 (2013) 015 [arXiv:1305.3139] [INSPIRE].

[48] R. Brustein and A.J.M. Medved, Phases of information release during black hole evaporation, JHEP 02 (2014) 116 [arXiv:1310.5861] [INSPIRE].

[49] R. Brustein and A.J.M. Medved, How black holes burn: Entanglement entropy evolution for an evaporating black hole, Phys. Rev. D 91 (2015) 084062 [arXiv:1407.4914] [INSPIRE].

[50] L. Alberte, R. Brustein, A. Khmelnitsky and A.J.M. Medved, Density matrix of black hole radiation, arXiv: 1502.02687 [INSPIRE].

[51] R. Brustein and A.J.M. Medved, Horizons of semiclassical black holes are cold, JHEP 06 (2014) 057 [arXiv: 1312.0880] [INSPIRE].

[52] R. Brustein and A.J.M. Medved, Black hole firewalls, smoke and mirrors, Phys. Rev. D 90 (2014) 024040 [arXiv: 1401.1401] [INSPIRE].

[53] R. Brustein and A.J.M. Medved, Constraints on the quantum state of pairs produced by semiclassical black holes, to appear.

[54] R. Brustein, Origin of the blackhole information paradox, Fortsch. Phys. 62 (2014) 255 [arXiv: 1209.2686] [INSPIRE].

[55] R. Brustein and J. Kupferman, Black hole entropy divergence and the uncertainty principle, Phys. Rev. D 83 (2011) 124014 [arXiv:1010.4157] [InSPIRE].

[56] R. Brustein and M. Hadad, Wave function of the quantum black hole, Phys. Lett. B 718 (2012) 653 [arXiv: 1202.5273] [INSPIRE].

[57] R. Brustein and A.J.M. Medved, Semiclassical black holes expose forbidden charges and censor divergent densities, JHEP 09 (2013) 108 [arXiv:1302.6086] [INSPIRE].

[58] D.N. Page, Average entropy of a subsystem, Phys. Rev. Lett. 71 (1993) 1291 [gr-qc/9305007] [INSPIRE].

[59] D.N. Page, Information in black hole radiation, Phys. Rev. Lett. 71 (1993) 3743 [hep-th/9306083] [INSPIRE].

[60] G. Dvali and C. Gomez, Black Hole's Quantum N-Portrait, Fortsch. Phys. 61 (2013) 742 [arXiv: 1112.3359] [INSPIRE].

[61] G. Dvali and C. Gomez, Black Hole's 1/N Hair, Phys. Lett. B 719 (2013) 419 [arXiv: 1203.6575] [INSPIRE].

[62] G. Dvali and C. Gomez, Black Holes as Critical Point of Quantum Phase Transition, Eur. Phys. J. C 74 (2014) 2752 [arXiv:1207.4059] [INSPIRE].

[63] G. Dvali and C. Gomez, Black Hole Macro-Quantumness, arXiv:1212.0765 [INSPIRE].

[64] G. Dvali, C. Gomez and D. Lüst, Black Hole Quantum Mechanics in the Presence of Species, Fortsch. Phys. 61 (2013) 768 [arXiv:1206.2365] [INSPIRE].

[65] P.C.W. Davies, S.A. Fulling and W.G. Unruh, Energy Momentum Tensor Near an Evaporating Black Hole, Phys. Rev. D 13 (1976) 2720 [INSPIRE]. 